\title{
MRNA structure determines specificity of a polyQ-driven phase separation
}

\author{
Erin M. Langdon, ${ }^{1}$ Yupeng Qiu, ${ }^{4}$ Amirhossein Ghanbari Niaki, ${ }^{4}$ Grace A. McLaughlin, ${ }^{1}$ Chase Weidmann, ${ }^{2}$ \\ Therese M. Gerbich,, Jean A. Smith, ${ }^{1}$ John M. Crutchley, ${ }^{1}$ Christina M. Termini, ${ }^{5}$ Kevin M. Weeks, ${ }^{2}$ Sua Myong, ${ }^{4}$ \\ Amy S. Gladfelter ${ }^{1,3^{*}}$ \\ ${ }^{1}$ Department of Biology, University of North Carolina at Chapel Hill, Chapel Hill, NC 27599, USA. ²Department of Chemistry, University of North Carolina at Chapel Hill, \\ Chapel Hill, NC 27599, USA. 'Marine Biological Laboratory, Woods Hole, MA 02543, USA. ${ }^{4}$ Department of Biophysics, Johns Hopkins University, Baltimore, MD 21218 , USA. \\ ${ }^{5}$ Division of Hematology/Oncology, Department of Medicine, University of California, Los Angeles, Los Angeles, CA 90095, USA. \\ *Corresponding author. Email: amyglad@unc.edu
}

RNA promotes liquid-liquid phase separation (LLPS) to build membraneless compartments in cells. How distinct molecular compositions are established and maintained in these liquid compartments is unknown. Here, we report that secondary structure allows mRNAs to self-associate and determines whether an mRNA is recruited to or excluded from liquid compartments. The polyQ-protein Whi 3 induces conformational changes in RNA structure and generates distinct molecular fluctuations depending on the RNA sequence. These data support a model in which structure-based, RNA-RNA interactions promote assembly of distinct droplets and protein-driven, conformational dynamics of the RNA maintain this identity. Thus, the shape of RNA can promote the formation and coexistence of the diverse array of RNArich liquid compartments found in a single cell.

Formation of non-membrane bound organelles through the condensation of macromolecules is a recently appreciated mechanism of intracellular organization. These liquid-like condensates form through liquid-liquid phase separation (LLPS) and are found in the cytoplasm and nucleus $(1,2)$. A fundamental unsolved problem is how liquid droplets recruit distinct constituents and retain independent identities. RNA can drive LLPS and modulates the material properties of droplets (3-6), but it is unknown if RNA controls the identity and maintenance of coexisting liquid compartments. Here we show mRNA secondary structure is required for droplet identity through directing interactions between mRNAs and RNA-binding proteins.

Whi3, a polyQ-containing, RNA-binding protein first identified in Saccharomyces cerevisiae (7), functions in morphogenesis, memory of mating, and stress responses, where it forms aggregates and associates with RNA-processing bodies (8-11). The homolog in the filamentous fungus Ashbya gossypii has one RNA recognition motif (RRM) and an expanded polyQ tract (fig. S1A), and both regions promote self-assembly. In vitro, Whi3 polyQ-dependent LLPS is driven by specific RNAs encoding regulators of either the cell cycle (e.g., the cyclin CLN3) or actin (e.g., the formin BNII and SPA2) (3). Distinct types of Whi3 droplets form in Ashbya cells: perinuclear CLN3 droplets and BNII droplets at sites of polarized growth at cell tips $(12,13)$ (Fig. 1A and movie S1). These two types of droplets have different Whi3 levels and Whi3 incorporation rates (Fig. 1, B and C), suggesting they are structurally distinct.
The distinct droplet properties may depend on extrinsic features of the local cytosolic microenvironment or arise due to different droplet constituents. CLN3 and BNI1 mRNAs minimally co-localize in the cytoplasm by single molecule (sm) RNA F.I.S.H., although they were occasionally co-expressed by the same nucleus (Fig. 1, D and F). The lack of colocalization suggests there are intrinsic, compositional differences between droplets. In contrast, mRNA of the polarity regulator $S P A 2$, frequently co-localized with $B N I 1$ mRNAs, especially at growth sites (Fig. 1, E and F). Thus, mRNAs encoding functionally related proteins co-localize, while functionally unrelated mRNAs do not. How can distinct Whi3-binding mRNAs segregate to different droplets in a common cytoplasm?

To address this question, we employed a reconstitution system to test if mRNA sequence was sufficient to generate droplet individuality (Fig. 2A). In vitro, as in cells, droplets composed of BNI1 mRNA displayed higher Whi3 to RNA molar ratios than droplets made with CLN3 mRNA (fig. S1B). Remarkably, when CLN3 mRNA was added to Whi3 droplets made with BNI1 mRNA, CLN3 preferentially assembled into new droplets, rather than incorporating into BNI1 droplets (Fig. 2, B and C, and fig. S1C). In contrast, BNI1 mRNA readily incorporated into preformed droplets (Fig. 2, B and C). Notably, SPA2 mRNA incorporated into BNII droplets (Fig. 2, B and C), and $C L N 3$ did not incorporate into $S P A 2$ droplets (fig. S1D). Thus, as in cells, cyclin and polarity mRNAs assemble into distinct and immiscible droplets in vitro, indicating droplet identity is encoded by the mRNA. 
mRNA sequences could influence droplet identity by favoring homotypic or specific heterotypic interactions between RNA molecules. To test for specific RNA-RNA interactions, we used a protein-free system to induce electrostatic-mediated phase transitions of the mRNA (14), where all mRNAs were capable of homotypic assembly into liquid or gel-like droplets (Fig. 2D). Strikingly, CLN3 mRNAs had minimal co-localization with $B N I 1$ or SPA2 mRNAs, whereas BNII and $S P A 2$ were significantly more co-localized (Fig. 2, E to G). Thus, sequence-encoded features of the mRNA can underpin the assembly of distinct, immiscible structures.

We next investigated which features of the mRNA sequence generate specificity. An mRNA with scrambled CLN3 coding sequence ( $c \ln 3 \mathrm{scr}$ ) with intact Whi3-binding sites formed Whi3 droplets (fig. S1E), but no longer showed specificity (Fig. 3, A and C). As the length, nucleotide composition, and Whi3 binding sites were identical, we hypothesized the secondary structure could promote specificity. CLN3 mRNA heated to $95^{\circ} \mathrm{C}$ to disrupt secondary structure also readily incorporated into Whi3-BNII droplets (Fig. 3, A and C). Melted $C L N 3$ mRNA that was slowly refolded (CLN3 refold) showed significantly less recruitment than melted, but more than native CLN3 (Fig. 3, A and C). Mixing between melted CLN3 and melted BNII occurred in the presence of Whi3 and in RNAonly reactions, suggesting mixing is initiated by RNA-RNA interactions (fig. S2). Thus, specificity information in CLN3 mRNA can be eliminated by disrupting secondary structure.

To identify what features of CLN3 mRNA secondary structure promote specificity, we performed SHAPE-MaP, which identifies highly flexible regions in RNA (15), to determine secondary structure changes, on native, refolded, and scrambled CLN3 mRNA (Fig. 3D and fig. S3, A and B). The first 400 nucleotides in the CLN3 sequence exhibited especially low SHAPE reactivity (fig. S3C, purple shaded regions), suggesting many paired nucleotides and a highly folded structure. Refolded CLN3 had a significant increase in SHAPE reactivity compared to native CLN3 (fig. S3A) $(P<0.001$, Wilcoxon rank sum test), indicating a transition to a more unstructured state (Fig. 3, D and E). Melting and refolding thus allows the RNA to sample different conformations from those formed during transcription. As expected, $c l n 3$ scr showed a different SHAPE profile with dramatically altered secondary structure (Fig. 3, D and E, and fig. S3B).

We hypothesized secondary structure influences mRNA sorting, as stem-loops may selectively display or mask sequences capable of hybridizing with other RNAs. CLN3 contains five complementary regions to BNII (fig. S4A), most of which had low SHAPE reactivity and therefore were more structured (fig. S4B), suggesting these regions are inaccessible for hybridizing with $B N I 1$. We hypothesize these regions became available to pair with BNI1 when CLN3 is melted, causing the structure-dependent loss of droplet specificity. To test this hypothesis, oligonucleotides (i.e., oligos) complementary to these regions were added to melted CLN3 and significantly decreased the co-assembly with $B N I 1$, restoring the formation of distinct $C L N 3$ droplets (Fig. 3, B and C). Additionally, $c \ln 3 \mathrm{sm}$, a mutant perturbing structure and exposing complementarity, co-localized with $B N I 1$ transcripts in vitro and at polarity sites in cells (Fig. $3 \mathrm{~F}$ and fig. S5) ( $>60 \%$ tips co-localized). Thus, secondary structure can regulate RNA sorting into distinct droplets through altering the capacity to form intermolecular interactions.

We next asked if exposed complementarity explains co-assembly of $B N I 1$ and $S P A 2$ into the same droplets. Indeed, SHAPE-MaP analysis of $B N I 1$ and $S P A 2$ showed complementary regions between these co-localizing mRNAs having significantly higher SHAPE reactivity and less structure compared to the $C L N 3 / B N I 1$ regions (figs. S4 and S6) $(P<$ $0.002, t$ test). Addition of complementary oligos to these regions disrupted co-localization in the presence of Whi3 and in RNA-only reactions (fig. S7, A and B). We predicted that CLN3 may self-assemble and indeed $\operatorname{cln} 3$ codon, a CLN3 mutant whose codons have been randomized but Whi3 binding sites remain intact, does not co-localize with endogenous CLN3 mRNA in cells, further supporting RNA-RNA interactions in co-assembly of related RNAs (fig. S7C). These data suggest RNA-RNA interactions based on intermolecular hybridization direct RNAs into the same or different droplets.

Does Whi3 protein influence the identity of droplets? The majority of Whi3 binding sites are exposed on stem loops in CLN3, BNI1, and SPA2 (Fig. 3E and figs. S8 and S9). Notably, refolding or scrambling the $C L N 3$ sequence rearranges the presentation of Whi3 binding sites (Fig. 3E). Therefore, RNA secondary structure may influence Whi3 binding and contribute to droplet composition and immiscibility in addition to RNA complexing. SHAPE-MaP of CLN3 mRNA in the presence of Whi3 support that Whi3 binding sites are occupied by protein (Fig. 4A and fig. S10A) and revealed that protein binding causes structural rearrangements (Fig. 4B). We therefore hypothesize Whi3 binding may have important contributions to structural rearrangements of target RNAs relevant to droplet identity.

To examine the consequence of Whi3 binding to RNA, we used smFRET (Fig. 4C) to measure the conformational dynamics of CLN3 and BNI1 mRNAs with and without Whi3 (16). In the absence of protein, CLN3 RNA showed high FRET values indicative of a compacted state, while BNI1 RNA showed lower FRET values, indicating a less compact state (Fig. 4D, purple shaded regions). Upon addition of Whi3, CLN3 FRET values decreased, indicating more extended RNA conformations were induced, dependent on the ability of Whi3 to bind mRNA (fig. S10, B and C). In contrast, bound to Whi3, BNI1 RNA showed a more substantial broadening of FRET values (Fig. 4D), indicating Whi3-BNI1 complexes are 
more dynamic. Dwell-time analysis revealed Whi3-induced dynamics are three times faster for BNI1 than CLN3 (Fig. 4E). Different mRNAs thus react differentially in their intramolecular fluctuations to the presence of Whi3, providing an additional mode of RNA droplet regulation.

These FRET studies suggest Whi3 binding alters the conformational dynamics of target RNAs. We speculate these differential dynamics help maintain droplet identities established by RNA-RNA interactions. Once RNA-RNA interactions are formed, Whi3 binding may reduce the ability of the RNA to resample many alternate RNA structures to maintain the identity. Additionally, the slower fluctuations of CLN3 bound to Whi3 may be one source of exclusion from the more rapidly fluctuating $B N I 1$-Whi3 complexes in those droplets. Such dynamics may drive the droplet material properties reported previously (3) and serve as barriers to homogenization.

We show mRNA structure defines the ability of an RNA to engage in homo- or heteromeric interactions and thus drives specificity in the composition of liquid droplet compartments (Fig. 4F). This mechanism is likely relevant for the sorting of specific RNAs to other RNA-granules such as stress and $\mathrm{P}$ granules, and P-bodies $(17,18)$. Future work will address the timing and location of how mRNA secondary structure influences selective uptake of cellular constituents into droplets. Protein binding to different RNAs can lead to varied dynamics of complexes, further distinguishing the physical properties of different droplets and promoting immiscibility of coexisting droplets. Given the large number of distinct, RNAbased condensates in the cell, these mechanisms are likely broadly relevant to explain how droplets achieve and maintain individuality.

\section{REFERENCES AND NOTES}

1. S. F. Banani, H. O. Lee, A. A. Hyman, M. K. Rosen, Biomolecular condensates: Organizers of cellular biochemistry. Nat. Rev. Mol. Cell Biol. 18, 285-298 (2017). doi:10.1038/nrm.2017.7 Medline

2. Y. Shin, C. P. Brangwynne, Liquid phase condensation in cell physiology and disease. Science 357, eaaf4382 (2017). doi:10.1126/science.aaf4382 Medline

3. H. Zhang, S. Elbaum-Garfinkle, E. M. Langdon, N. Taylor, P. Occhipinti, A. A. Bridges, C. P. Brangwynne, A. S. Gladfelter, RNA controls PolyQ protein phase transitions. Mol. Cell 60, 220-230 (2015). doi:10.1016/i.molcel.2015.09.017 Medline

4. X. Zhang, Y. Lin, N. A. Eschmann, H. Zhou, J. N. Rauch, I. Hernandez, E. Guzman, K. S. Kosik, S. Han, RNA stores tau reversibly in complex coacervates. PLOS Biol. 15 e2002183 (2017). doi:10.1371/journal.pbio.2002183 Medline

5. S. Elbaum-Garfinkle, Y. Kim, K. Szczepaniak, C. C.-H. Chen, C. R. Eckmann, S. Myong, C. P. Brangwynne, The disordered P granule protein LAF-1 drives phase separation into droplets with tunable viscosity and dynamics. Proc. Natl. Acad. Sci. U.S.A. 112, 7189-7194 (2015). doi:10.1073/pnas.1504822112 Medline

6. Y. Lin, D. S. W. Protter, M. K. Rosen, R. Parker, Formation and maturation of phaseseparated liquid droplets by RNA-binding proteins. Mol. Cell 60, 208-219 (2015). doi:10.1016/i.molcel.2015.08.018 Medline

7. R. S. Nash, T. Volpe, B. Futcher, Isolation and characterization of WHI3, a sizecontrol gene of Saccharomyces cerevisiae. Genetics 157, 1469-1480 (2001). Medline

8. N. Colomina, F. Ferrezuelo, E. Vergés, M. Aldea, E. Garí, Whi3 regulates morphogenesis in budding yeast by enhancing Cdk functions in apical growth. Cell Cycle 8, 1912-1920 (2009). doi:10.4161/cc.8.12.8740 Medline

9. F. Caudron, Y. Barral, A super-assembly of Whi3 encodes memory of deceptive encounters by single cells during yeast courtship. Cell 155, 1244-1257 (2013). doi:10.1016/i.cell.2013.10.046 Medline

10. G. Schlissel, M. K. Krzyzanowski, F. Caudron, Y. Barral, J. Rine, Aggregation of the Whi3 protein, not loss of heterochromatin, causes sterility in old yeast cells. Science 355, 1184-1187 (2017). Medline

11. K. J. Holmes, D. M. Klass, E. L. Guiney, M. S. Cyert, Whi3, an S. cerevisiae RNAbinding protein, is a component of stress granules that regulates levels of its target mRNAs. PLOS ONE 8, e84060 (2013). doi:10.1371/journal.pone.0084060 Medline

12. C. Lee, H. Zhang, A. E. Baker, P. Occhipinti, M. E. Borsuk, A. S. Gladfelter, Protein aggregation behavior regulates cyclin transcript localization and cell-cycle control. Dev. Cell 25, 572-584 (2013). Medline

13. C. Lee, P. Occhipinti, A. S. Gladfelter, PolyQ-dependent RNA-protein assemblies control symmetry breaking. J. Cell Biol. 208, 533-544 (2015). doi:10.1083/jacb.201407105 Medline

14. A. Jain, R. D. Vale, RNA phase transitions in repeat expansion disorders. Nature 546, 243-247 (2017). doi:10.1038/nature22386 Medline

15. M. J. Smola, G. M. Rice, S. Busan, N. A. Siegfried, K. M. Weeks, Selective 2'-hydroxyl acylation analyzed by primer extension and mutational profiling (SHAPE-MaP) for direct, versatile and accurate RNA structure analysis. Nat. Protoc. 10, 1643-1669 (2015). doi:10.1038/nprot.2015.103 Medline

16. Y. Kim, S. Myong, RNA remodeling activity of DEAD box proteins tuned by protein concentration, RNA Length, and ATP. Mol. Cell 63, 865-876 (2016). doi:10.1016/j.molcel.2016.07.010 Medline

17. T. Trcek, M. Grosch, A. York, H. Shroff, T. Lionnet, R. Lehmann, Drosophila germ granules are structured and contain homotypic mRNA clusters. Nat. Commun. 6 , 7962 (2015). doi:10.1038/ncomms8962 Medline

18. B. Van Treeck, D. S. W. Protter, T. Matheny, A. Khong, C. D. Link, R. Parker, RNA self-assembly contributes to stress granule formation and defining the stress granule transcriptome. Proc. Natl. Acad. Sci. U.S.A. 115, 2734-2739 (2018). Medline

19. J. Schindelin, I. Arganda-Carreras, E. Frise, V. Kaynig, M. Longair, T. Pietzsch, S. Preibisch, C. Rueden, S. Saalfeld, B. Schmid, J.-Y. Tinevez, D. J. White, V. Hartenstein, K. Eliceiri, P. Tomancak, A. Cardona, Fiji: An open-source platform for biological-image analysis. Nat. Methods 9, 676-682 (2012) doi:10.1038/nmeth.2019 Medline

20. MATLAB and Statistical Toolbox Release R2017b (The MathWorks, Inc., Natick, MA, USA; https://www. mathworks.com/products/matlab.html).

21. R: A Language and Environment for Statistical Computing ( $\mathrm{R}$ Core Team, $\mathrm{R}$ Foundation for Statistical Computing, Vienna, Austria, 2017; https://www.Rproject.org.

22. S. Busan, K. M. Weeks, Accurate detection of chemical modifications in RNA by mutational profiling (MaP) with Shapemapper 2. RNA 24, 143-148 (2017). Medline

\section{ACKNOWLEDGMENTS}

We thank the Gladfelter, Weeks, and Laederach labs for critical discussions, Drs Griffin, Moseley, Lew, Peifer, and Higgs for critically reading the manuscript, the $\mathrm{HHMI} \mathrm{HCIA} \mathrm{at} \mathrm{the} \mathrm{Marine} \mathrm{Biological} \mathrm{Laboratory} \mathrm{for} \mathrm{intellectual} \mathrm{community,} \mathrm{and}$ T. Straub for useful data analysis discussions. Funding: This work was supported by NIH GM R01-GM081506, the HHMI Faculty Scholars program, R35 GM122532, ACS 130845-RSG-17-114-01-RMC, NIH 1DP2 GM105453, and NIH R01 GM115631. Author contributions: E.M.L. and A.S.G. designed and performed experiments, analyzed data, prepared figures, and drafted the manuscript; P.B., A.G.N., and C.W. designed and performed experiments, analyzed data, and edited the manuscript; G.A.M. and C.M.T. performed experiments and analyzed data; T.M.G., J.A.S., and J.M.C. provided technical support and edited the manuscript; K.M.W. and S.M. designed experiments and edited the manuscript. Competing interests: K.M.W. is an advisor to and holds equity in Ribometrix, to which mutational profiling $(\mathrm{MaP})$ technologies have been licensed. All other authors declare that they have no competing interests. Data 
and materials availability: All data are available upon request from E.M.L. or A.S.G.

\section{SUPPLEMENTARY MATERIALS}

www.sciencemag.org/cgi/content/full/science.aar7432/DC1

Materials and Methods

Figs. S1 to S10

Movie S1

References (19-22)

12 December 2017; accepted 4 April 2018

Published online 12 April 2018

10.1126/science.aar7432 

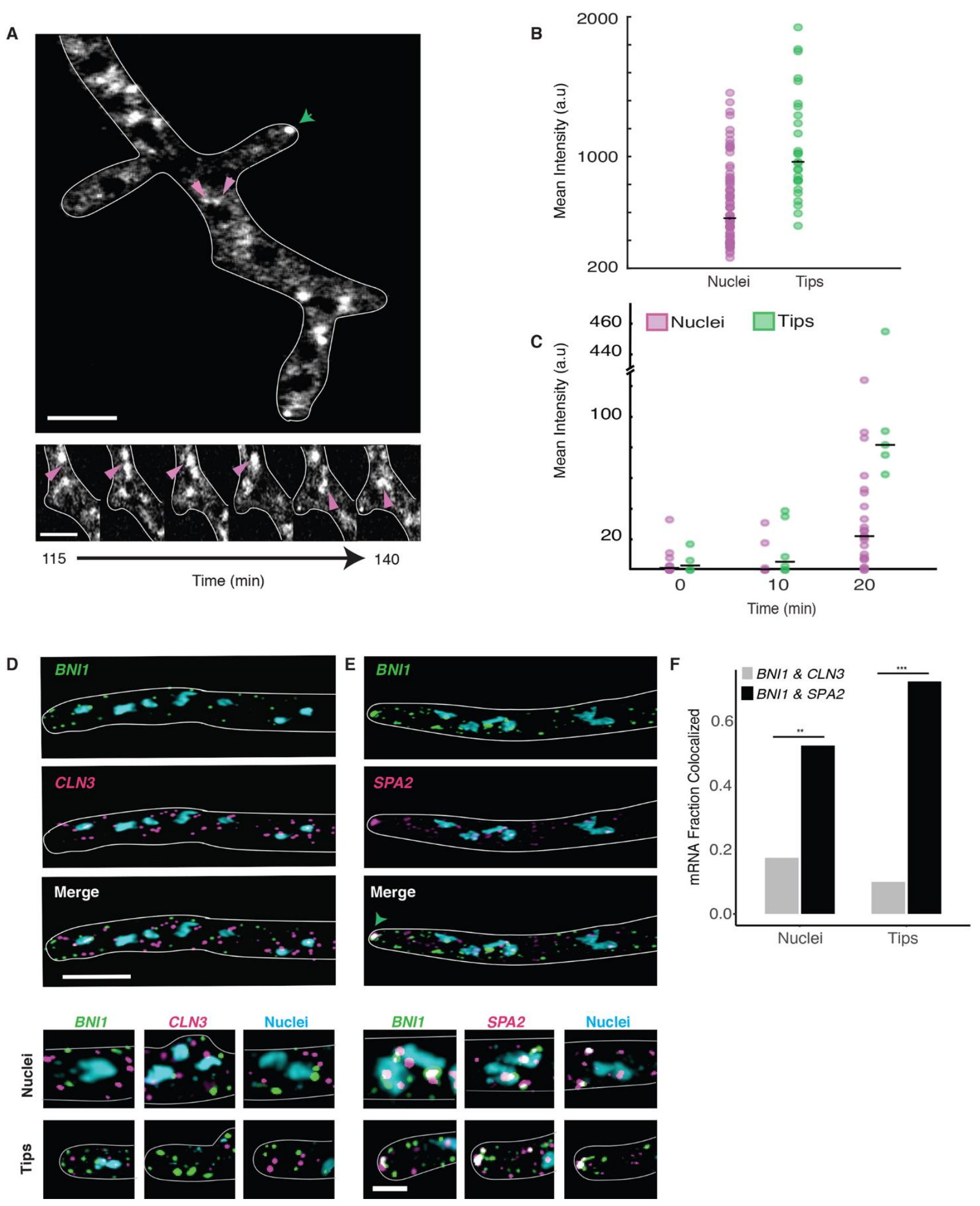
Fig. 1. Cyclin and polarity complexes are spatially and physically distinct within the cell. (A) Top, Whi3 forms liquid droplets in Ashbya gossypii. Below, Whi3 droplets accumulate and fuse around nuclei. Green arrows denote polarity droplets. Pink arrows denote perinuclear droplets. Scale bars $5 \mu \mathrm{m}$. (B) Mean intensity of Whi3-tomato is higher in tip droplets (green) than perinuclear droplets (pink). (C) Rate of Whi3 incorporation is higher in tip compared to perinuclear droplets. (D) smFISH images show BNII (green) and CLN3 (pink) mRNAs are spatially distinct. Nuclei are in blue. Scale bar $5 \mu \mathrm{m}$. (E) smFISH images show BNII (green) mRNAs co-localize with polarity mRNA SPA2 (pink). Nuclei are in blue. The green arrow marks where the RNAs overlap at the tip. Inset scale bar $2 \mu \mathrm{m}$. (F) BNI1 and SPA2 are significantly more colocalized than BNI1 and CLN3. $P<0.001$ for tips and $P<0.01$ for nuclei (Fisher's Exact test). $n=40$ nuclei and tips for $\geq 30$ cells. 
A BNI1 + Whi3 Cy3 labeled mRNA $\begin{gathered}\text { mRNA not sufficient } \\ \text { for segregation }\end{gathered}$ droplet

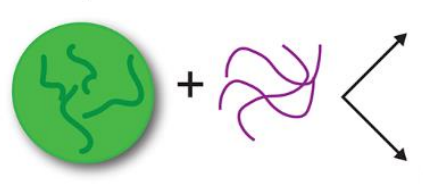

mRNA sufficient for segregation

c
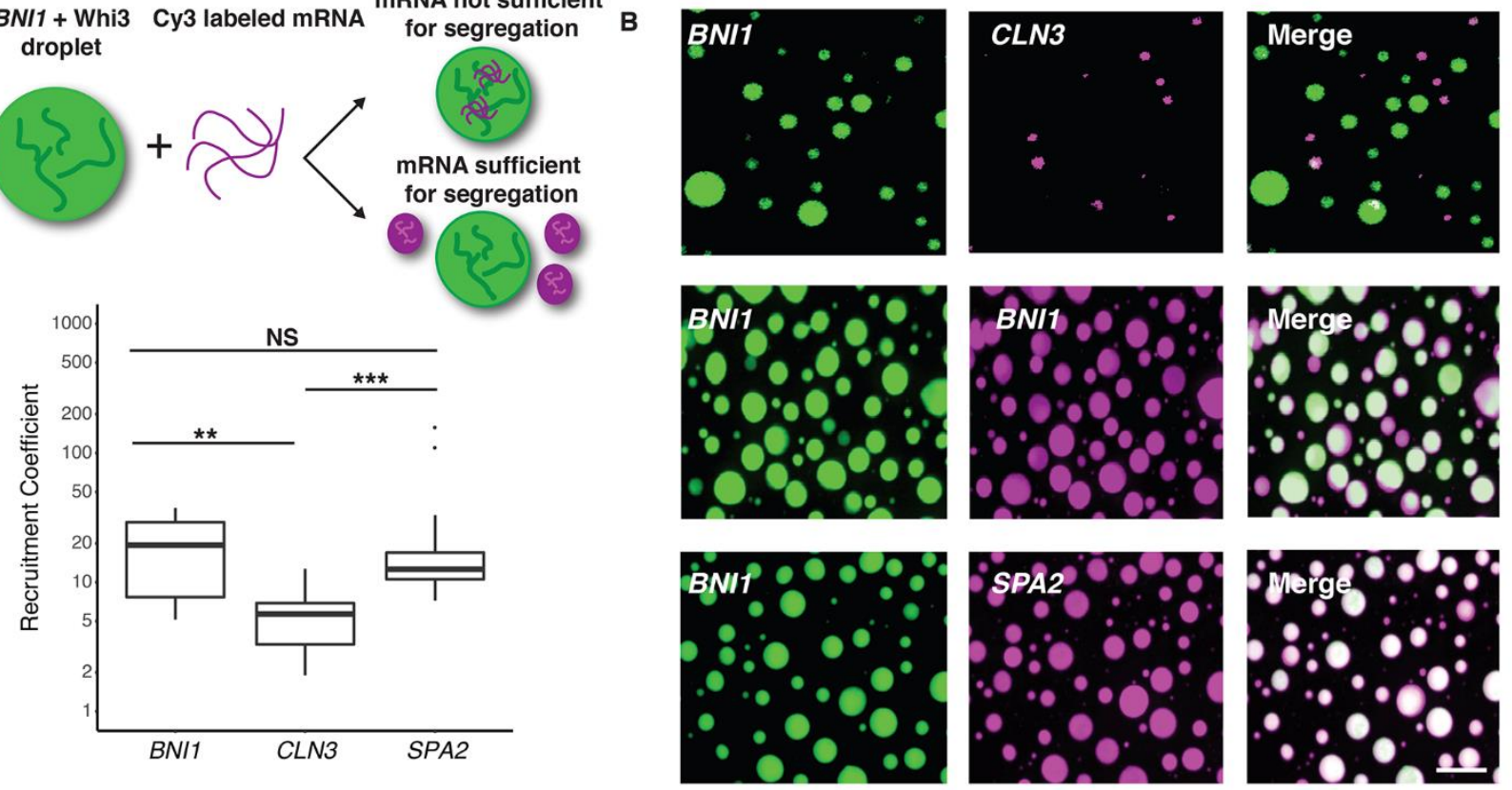

D
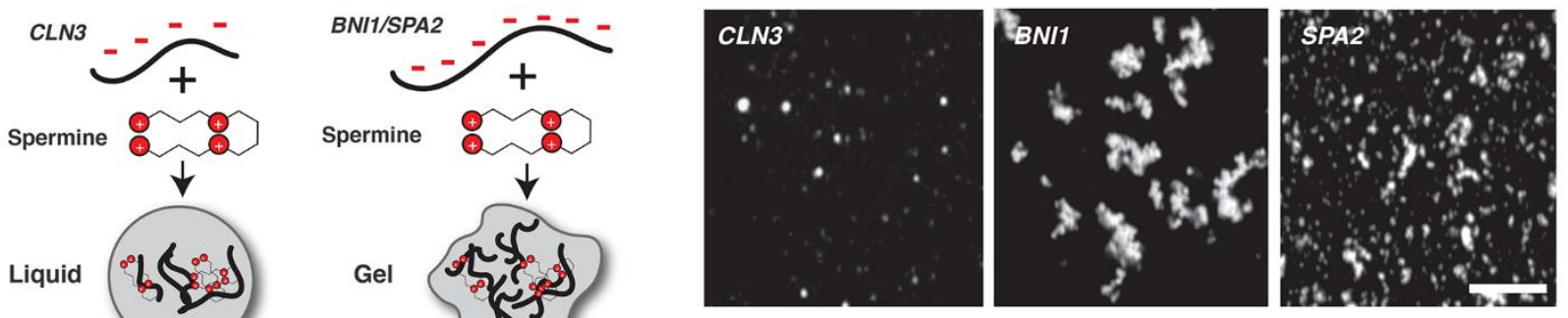

E

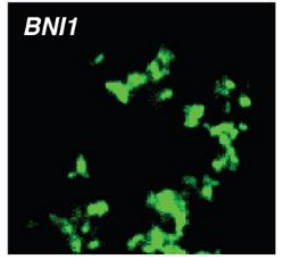

F
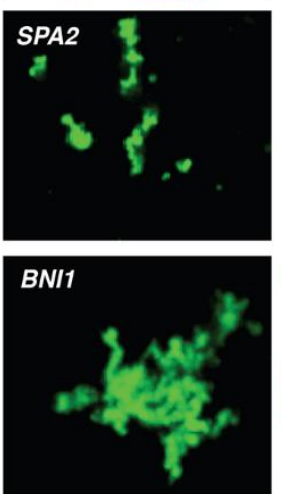
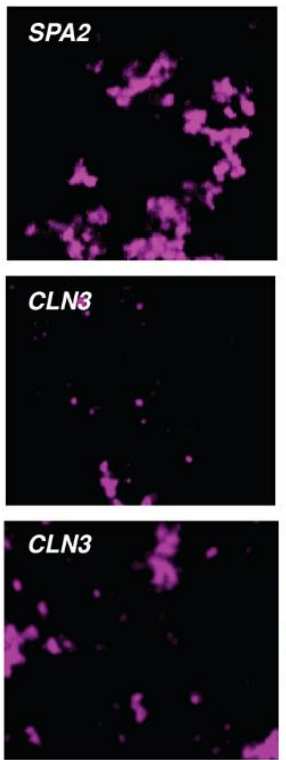
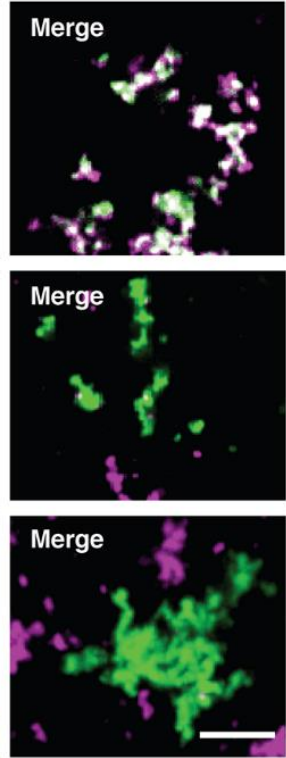

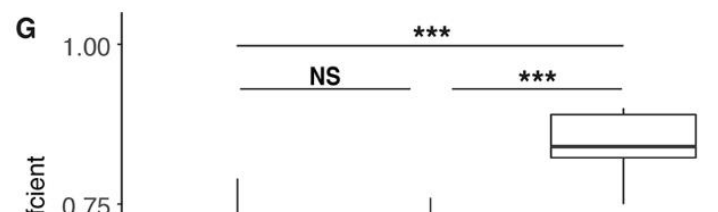

$C L N 3+S P A 2 \quad C L N 3+B N / 1 \quad B N / 1+S P A 2$ 
Fig. 2. Polarity and cyclin complexes segregate in vitro. (A) Experimental schematic of in vitro droplet recruitment assay. (B) CLN3 mRNA (pink) is not efficiently recruited but BNI1 or SPA2 mRNA (pink) are recruited into preformed Whi3-BNI1 droplets (green) based on fluorescence microscopy. Scale bar 10 $\mu \mathrm{m}$. (C) Recruitment coefficients of mRNA from (B). Boxes indicate interquartile range, line is median and whiskers contain points within three times the interquartile range, and outliers are indicated with $(\bullet)$ marks. NS, not significant, $P>0.05$; **, $P<0.01$; ***, $P<0.001$ ( $t$ test). $n \geq 500$ droplets for $N \geq 3$ biological replicates. (D) Cartoon schematic and representative images showing in vitro RNA-only droplet assay where $C L N 3, B N I 1$, and SPA2 mRNAs assemble into liquid or gel-like droplets. Scale bar 5 $\mu \mathrm{m}$. (E) Fluorescence microscopy images showing BNI1 RNA (green) colocalizes with SPA2 RNA (pink) in droplets. (F) Fluorescence microscopy images showing CLN3 RNA (pink) does not colocalize with SPA2 (green) and BNII (green) droplets. Scale bar $5 \mu \mathrm{m}$. (G) Quantification of co-localization between BNI1 and SPA2, SPA2 and CLN3, or BNI1 and CLN3 RNAs. NS, not significant, ${ }^{* *}, P<0.001$ (Wilcoxon rank-sum test). $n \geq 200$ droplets for $N \geq 3$ biological replicates. 
A
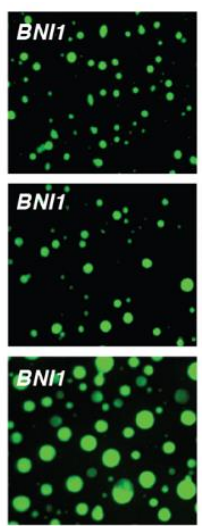

B

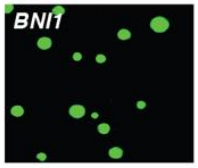

D
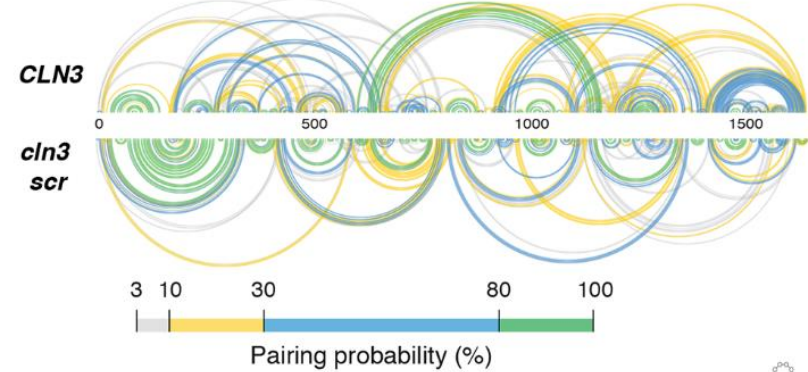

E

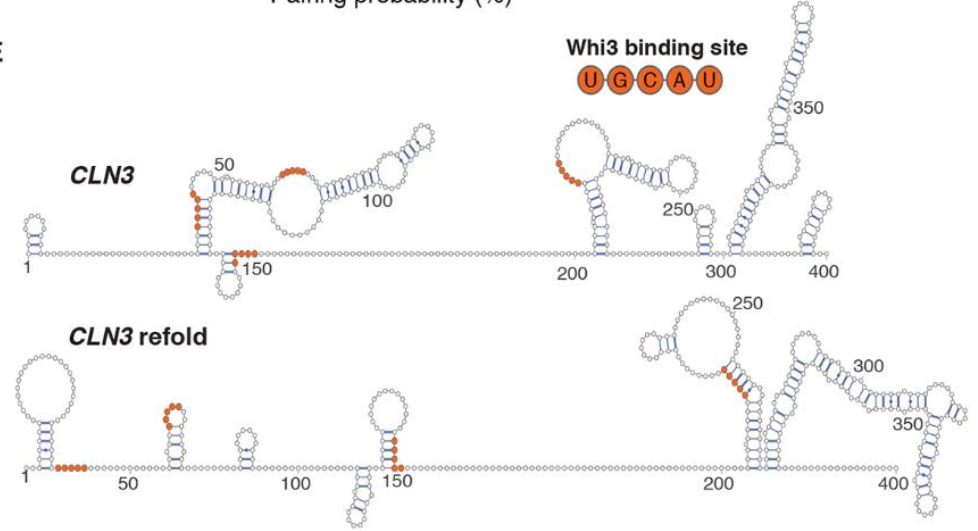

cln3 scr

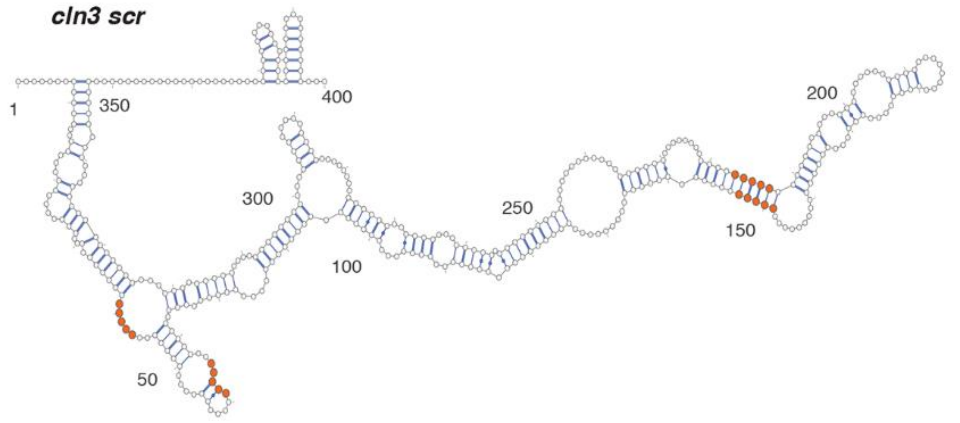

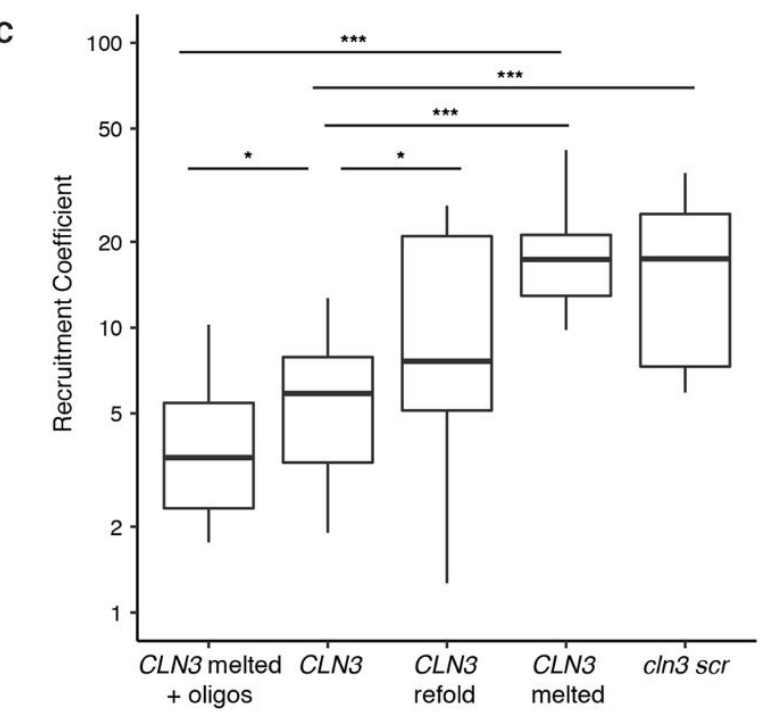

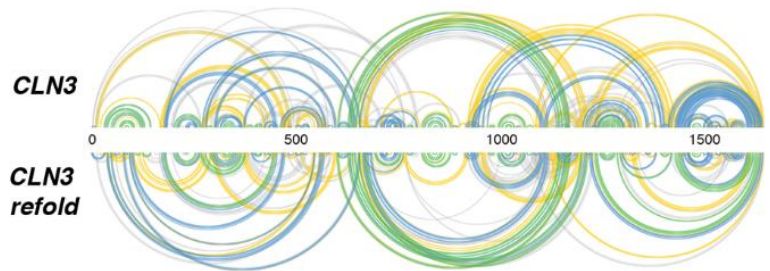

F
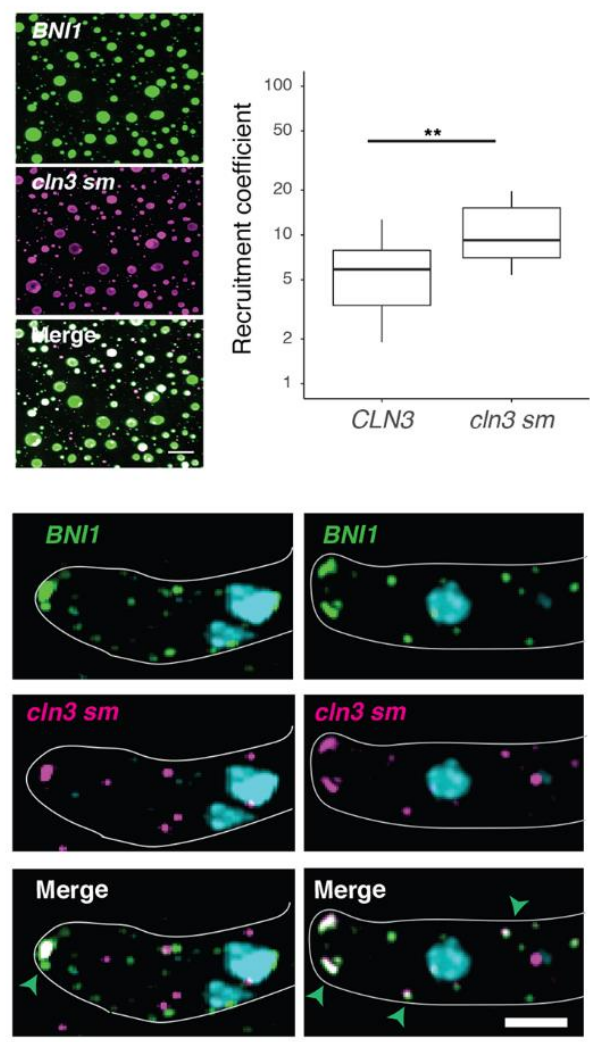
Fig. 3. RNA secondary structure determines specificity and identity of Whi3-CLN3 droplets. (A) Fluorescence microscopy images showing the recruitment of scrambled (cln3 scr), melted (CLN3 ml), and refolded CLN3 (CLN3 refold) mRNA (pink) into preformed Whi3-BNI1 droplets (green). (B) Fluorescence microscopy images showing the loss of recruitment of CLN3 ml when mixed with oligonucleotides targeting complementary sequences of CLN3 to BNI1. Scale bar 10 $\mu \mathrm{m}$. (C) Quantification of (A) and (B). * $P<0.05$; ${ }^{*}, P<0.01$; ${ }^{* * *}, P<0.001$ ( $t$ test). $n \geq 500$ droplets for $N \geq 3$ biological replicates. (D) Base pairing probability from SHAPE-MaP of CLN3, $\mathrm{cln} 3 \mathrm{scr}$, and $C L N 3$ refold show differences in the secondary structure in CLN3. Arcs connect base pairs and are colored by probability. (E) Secondary structure models from SHAPE-MaP for the first 400 nucleotides of $C L N 3, C L N 3$ refold, and $c / n 3 \mathrm{scr}$. Whi3 binding sites are in orange. $(\mathrm{F}) \mathrm{CLN} 3$ structure mutant ( $\mathrm{cln} 3 \mathrm{sm}$ ) mRNA is significantly recruited to Whi3-BNII droplets in vitro and in vivo. ${ }^{*}, P<0.01$ ( $t$ test). Green arrows denote sites of co-localization between BNI1 mRNA (green) and $\operatorname{cln} 3 \mathrm{sm}$ mRNA (pink) by smFISH. Scale bar $10 \mu \mathrm{m}$ for in vitro, $2 \mu \mathrm{m}$ for in vivo. $n \geq 500$ droplets for $N \geq 3$ biological replicates. 
A

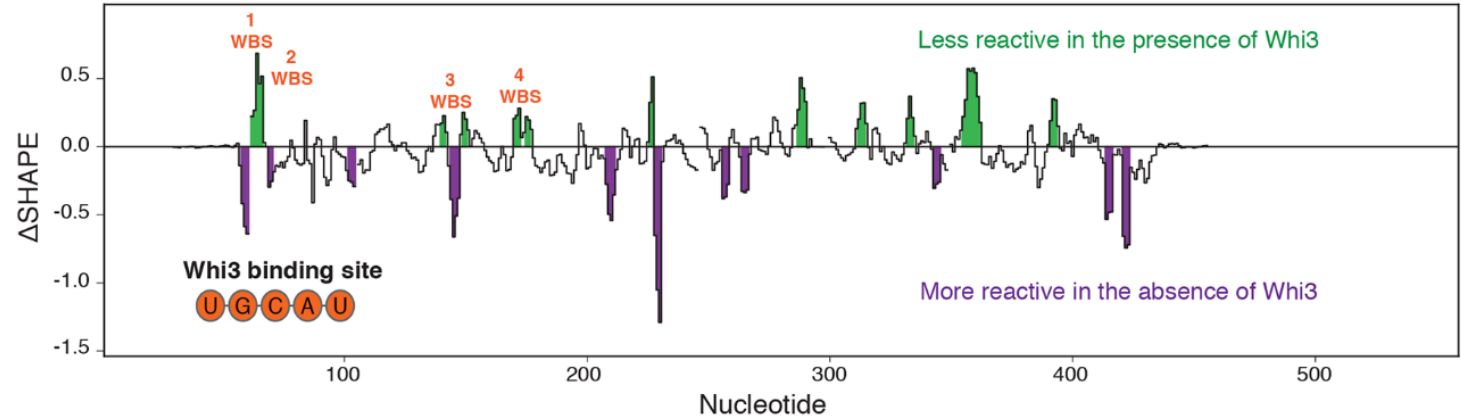

B

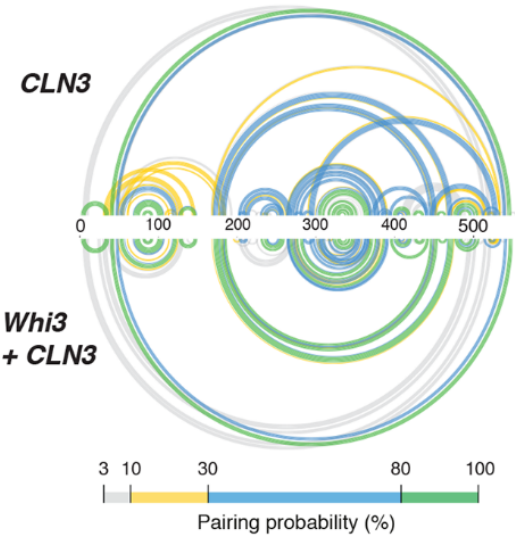

E

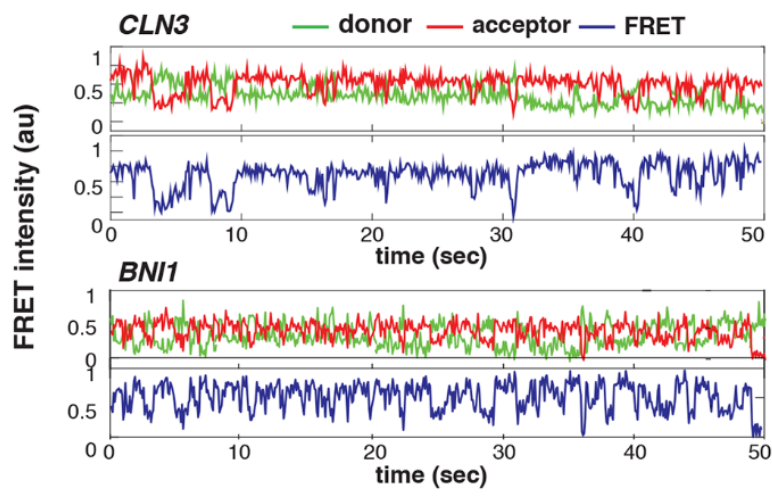

C $\quad$ donor

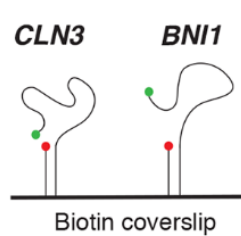

Biotin coverslip
D
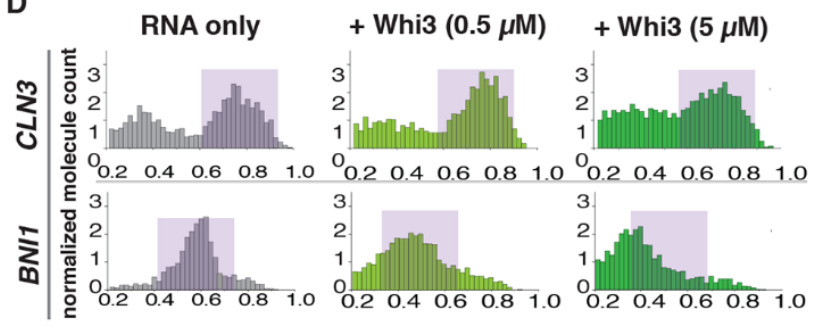

FRET

$\mathbf{F}$
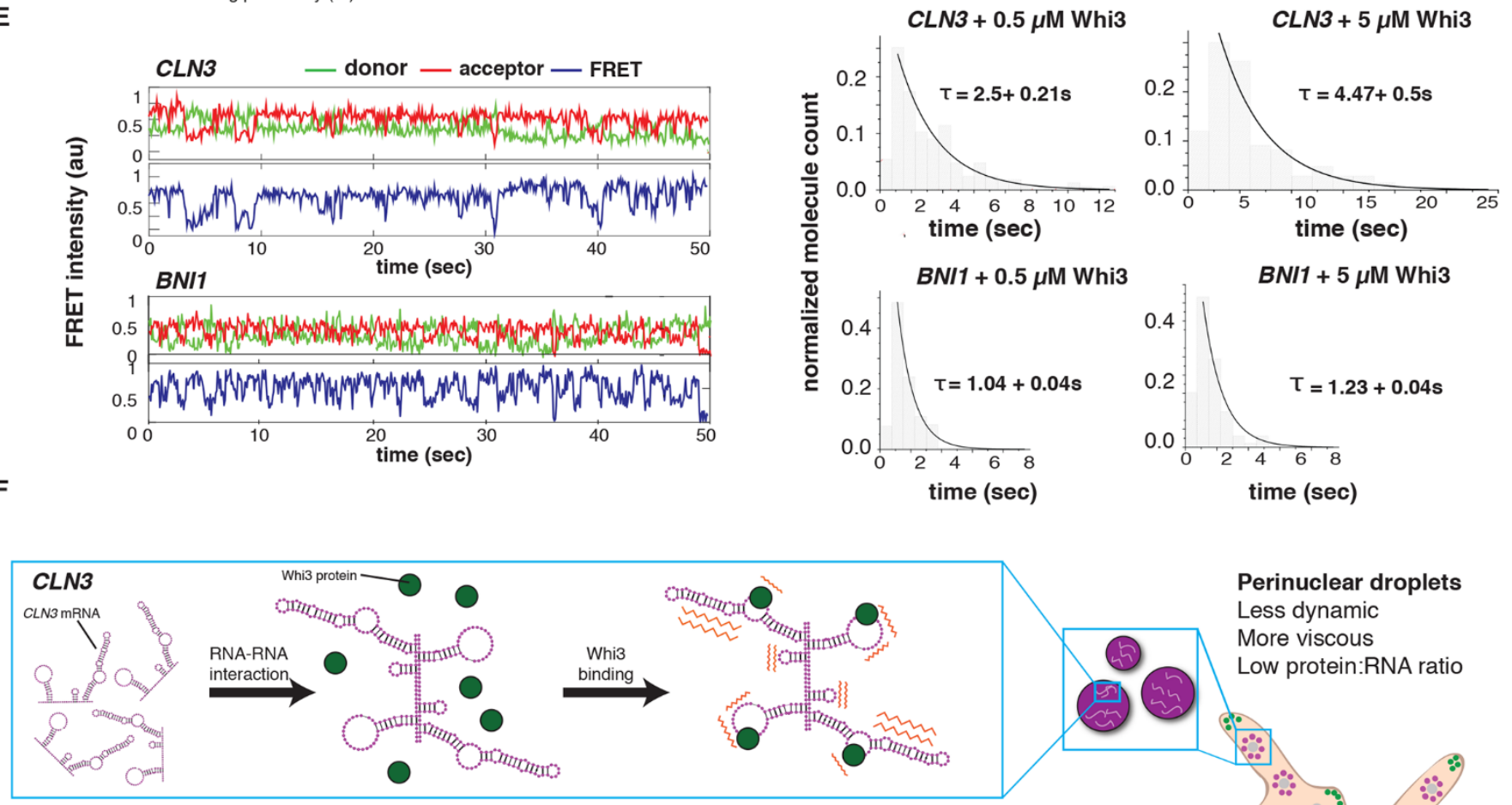

Perinuclear droplets Less dynamic

More viscous

Low protein:RNA ratio
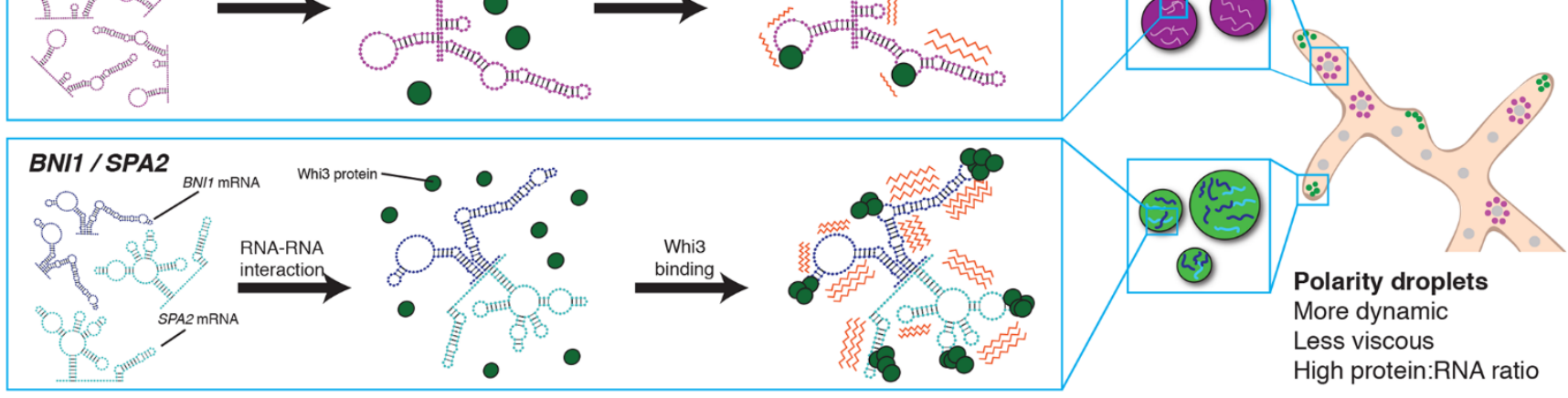
Fig. 4. Whi3 binding alters RNA behavior. (A) Differences in SHAPE reactivities ( $\triangle S H A P E$ ) were calculated by subtracting CLN3 SHAPE reactivities from CLN3 + Whi3 reactivities. Positive $\triangle$ SHAPE values indicate protection from modification in the presence of Whi3 and negative $\triangle$ SHAPE reports enhanced reactivity in the absence of Whi3 protein. (B) Base pairing probability compared between CLN3 and CLN3 with Whi3 shows rearrangements in CLN3 structure in the presence of Whi3. Arcs connect base pairing sites and are colored by probability. (C) Schematic of smFRET experiment. (D) FRET histograms before (gray) and after (green) 0.5 or $5 \mu \mathrm{M}$ Whi3 addition. Purple shaded regions denote high and mid FRET states for CLN3 and BNII, respectively. (E) Averaged cy3 (green), cy5 (red) intensities, and representative FRET traces (blue) obtained from smFRET experiments of CLN3 and BNI1 in the presence of $5 \mu \mathrm{M}$ Whi3. Dwell time analysis reveals slower FRET fluctuations for CLN3 than $B N I 1$ in the presence of Whi3. (F) Proposed model in which RNA-RNA interactions derived from mRNA structure promotes the selective uptake of distinct RNAs and protein constituents into droplets leading to distinct dynamics (orange zigzags) of different droplet complexes. 